\title{
Electronically Controllable Current-Mode Multiphase Sinusoidal Oscillator for Biomedical Tissue Measurement Systems
}

\author{
S. Siripongdee, P. Suwanjan, S. Tuntrakool, and W. Jaikla
}

\begin{abstract}
The design of current-mode multiphase sinusoidal oscillator (MSO) for biomedical tissue measurement system is presented. The odd phase system can be realised using current controlled current differencing transconductance amplifier (CCCDTA)-based lossy integrators. The condition of oscillation and frequency of oscillation can be controlled electronically and independently through adjusting the current of the CCCDTA. The high output impedances facilitate easy driving an external load without additional current buffers. The proposed MSO provides odd phase signals that are equally spaced in phase and equal amplitude. The circuit requires one CCCDTA and one grounded capacitor per phase without external resistor and additional current amplifier. The results of PSPICE simulations using BJT CCCDTA are included to verify theory.
\end{abstract}

Index Terms-CCCDTA, multiphase sinusoidal oscillator, integrated circuit.

\section{INTRODUCTION}

Multiphase sinusoidal oscillator (MSO) is important blocks for various applications. For example, in telecommunications it is used for phase modulators, quadrature mixers [1], and single-sideband generators [2]. In measurement system, MSO is employed for vector generator or selective voltmeters [3]. It can also be utilized in power electronics systems [4]. Recently, current-mode circuits have been receiving considerable attention of due to their potential advantages such as inherently wide bandwidth, lower slew-rate, greater linearity, wider dynamic range, simple circuitry and low power consumption [5]. Many active building blocks (ABBs) have been proposed to realize the current-mode circuit. The interesting active element, called current controlled current differencing transconductance amplifier (CCCDTA) [6], [7], is introduced to provide new possibilities in the current-mode circuit. It is really current-mode element whose input and output signal are currents. In addition, output currents of CCCDTA can be electronically adjusted.

Several realizations of current-mode MSOs using different active building blocks are available in the literature. These include realizations using current follower (CF) [8], CCCII [9]-[11], CDTA [12]-[14], CDBA [15], CFOA [16], and CCCCTA [17] and CCCDTA [18], [19]. The CF-based MSO

Manuscript received September 17, 2013; revised November 21, 2013 This work was supported in part by Faculty of Industrial Education, King Mongkut's Institute of Technology Ladkrabang (KMITL) under Grant 25570203021.

The authors are with Department of Engineering Education, Faculty of Industrial Education, King Mongkut's Institute of Technology Ladkrabang, Bangkok, Thailand (e-mail: kssurapo@kmitl.ac.th, kspeeraw@kmitl.ac.th, ktsunti@kmitl.ac.th, kawinai@kmitl.ac.th). in [8] requires two current followers, one floating resistor, and one floating capacitor for each phase and thus the circuit is not suitable for monolithic integration. Moreover, it cannot be electronically controlled. The CCCII-based MSOs [9]-[11] enjoy high-output impedances and electronic tunability. However, the first one requires a large number of external capacitors. In addition, the oscillation condition can be provided by tuning the capacitance ratio of external capacitors, which is not easy to implement. The second reported circuit requires additional current amplifiers, which makes the circuit more complicated and increases its power consumption. CDTA-based current-mode MSOs in [12] is based on lossy integrators, where as the circuits in [13] and [14] contain CDTA-based allpass sections. They exhibit good performance in terms of electronic tunability, high-output impedances, and independent control of the oscillation frequency and the oscillation condition. However, MSOs in [12], [13] require an additional current amplifier, which is implemented by two CDTAs. Moreover, the output currents of the MSO, utilizing the CDTA-based lossy integrators, are of different amplitudes. The MSO employing CDTA-based allpass sections [13] requires two CDTAs in each allpass section, and the circuitry becomes more extensive. While MSO using CDTA-based allpass sections [14] requires floating capacitor. Consequently, it occupies a larger chip area for VLSI design. In addition, its power consumption is also increased.

The purpose of this study is to introduce a new current-mode multiphase sinusoidal oscillator. The features of the proposed circuit are the following: 1) Use of grounded capacitors and identical circuit configuration for each section in the MSO topology which are suitable for integration. 2) The electronic tunability of oscillation condition and oscillation frequency. 3) High-impedance current outputs. 4) The possibility of generating multi-phase signals for both an even and odd number of equally-spaced in phases. 5) Independent tuning of the oscillation frequency and the oscillation condition. 6) Equality of amplitudes of each phase due to utilizing identical sections. 7) Requirement for only one CCCDTA as the active element for each phase without any additional current amplifiers.

\section{Proposed Multiphase Sinusoidal Oscillator}

The main active element used to design the proposed inductance simulator is CCCDTA. Thus, the review of it will be shown here. The principle of CCCDTA was introduced in [7]. Its symbol and equivalent circuit are shown respectively in Fig. 1 a) and Fig. 1 b). The $\mathrm{p}$ and $\mathrm{n}$ which have finite resistances $\left(R_{p}\right.$ and $\left.R_{n}\right)$ are the current input terminals. $z$ and $x$ 
are the output terminals. The difference of input currents $\left(i_{p}-i_{n}\right)$ will send to $z$ terminal. The voltage at $z$ terminal is converted to the $x$-terminal current via a transconductance $g_{m}$. The characteristics of CCCDTA can be described by:

$$
\left[\begin{array}{l}
V_{p} \\
V_{n} \\
I_{z} \\
I_{x}
\end{array}\right]=\left[\begin{array}{cccc}
R_{p} & 0 & 0 & 0 \\
0 & R_{n} & 0 & 0 \\
1 & -1 & 0 & 0 \\
0 & 0 & 0 & \pm g_{m}
\end{array}\right]\left[\begin{array}{l}
I_{p} \\
I_{n} \\
V_{x} \\
V_{z}
\end{array}\right]
$$

If the CCCDTA is realized using BJT technology, $R_{p}, R_{n}$ and gm can be written as

$$
R_{p}=R_{n}=\frac{V_{T}}{2 I_{B 1}}
$$

and

$$
g_{m}=\frac{I_{B 2}}{2 V_{T}}
$$

Here $V_{T}$ is the thermal voltage. $I_{B 1}$ and $I_{B 2}$ are the bias currents used to control the intrinsic resistances and transconductance, respectively. The internal construction of BJT CCCDTA is shown in Fig. 2.
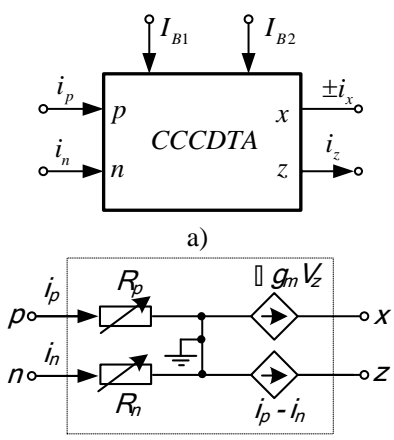

b)

Fig. 1 a) Electrical circuit symbol and b) Equivalent circuit of CCCDTA

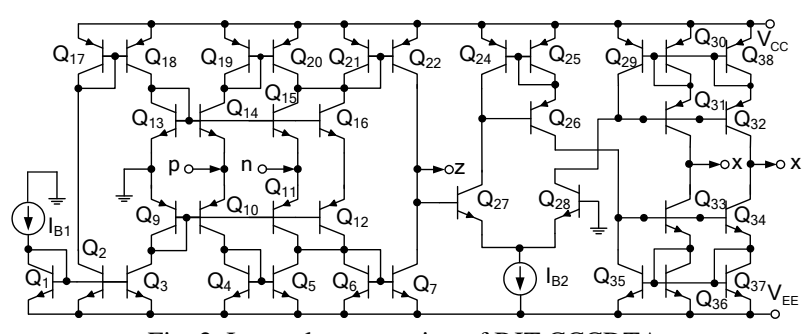

Fig. 2. Internal construction of BJT CCCDTA.

The generalized structure of MSO is designed by cascading the $n$ identical stages $(n \geq 3)$ which contains the lossy integrator (first order low pass filter) for each phase. The output of $n^{\text {th }}$ stage is fed back to the input of the first stage. The system can provide one phase per one lossy integrator without any additional external amplifier. The current-mode odd phase MSO is shown in Fig. 3. It is found from Fig. 3 that the current mirrors are required to split the bias currents $I_{B 1}$ and $I_{B 2}$ to each lossy integrator section. In addition, it can be seen that the proposed MSO enjoy highoutput impedances which facilitate easy driving an external load without additional current buffers. From circuit in Fig. 3 for $n=3,5,7, \ldots$, the frequency of oscillation (FO) and condition of oscillation (CO) are expressed as [16]

$$
F O: \omega_{o s c}=\frac{1}{R_{p} C} \tan \frac{\pi}{n}
$$

and

$$
C O: \frac{g_{m} R_{n}}{2} \geq \sec \frac{\pi}{n}
$$

From Eqs. (4) and (5), if $R_{p}=R_{n}=V_{T} / 2 I_{B 1}$ and $g_{m}=I_{B 2} / 2 V_{T}$, the $\mathrm{FO}$ and $\mathrm{CO}$ is modified as

$$
\omega_{\text {osc }}=\frac{2 I_{B 1}}{V_{T} C} \tan \frac{\pi}{n}
$$

and

$$
\frac{I_{B 2}}{8 I_{B 1}} \geq \sec \frac{\pi}{n}
$$

From Eqs. (6) and (7), it can be seen that the $\mathrm{CO}$ can be adjusted electronically/independently from the FO by varying $I_{B 2}$.

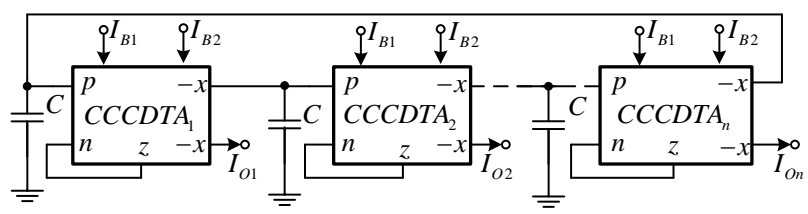

Fig. 3. Proposed MSO.

\section{Simulation Results}

To prove the performances of the proposed MSO, the PSpice simulation program was used for the examination. The PNP and NPN transistors employed in the proposed circuit were simulated by using the parameters of the PR200N and NR200N bipolar transistors of ALA400 transistor array from AT\&T [20]. The CCCDTA has been simulated using the bipolar technology structure of Fig. 2. The circuit was biased with $\pm 3 \mathrm{~V}$ supply voltages.

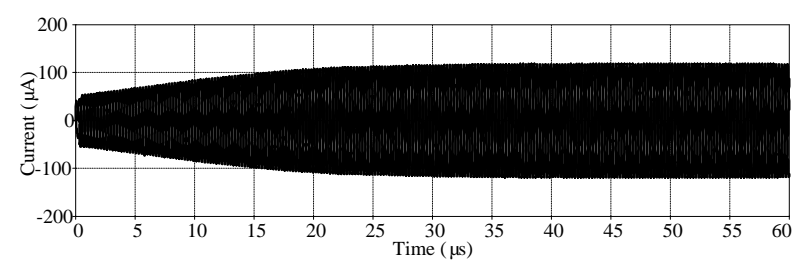

Fig. 4. Sinusoidal output currents at initial time.

An odd three-phase sinusoidal oscillator $(n=3)$ based on the structure in Fig. 3 has been designed. The component values are as follows: $I_{B 1}=40 \mu \mathrm{A}, I_{B 2}=630 \mu \mathrm{A}, C=0.5 \mathrm{nF}$. The simulated output waveforms, $\mathrm{I}_{\mathrm{O} 1}, \mathrm{I}_{\mathrm{O} 2}$ and $\mathrm{I}_{\mathrm{O} 3}$ are shown in Fig. 4 and Fig. 5. The frequency of oscillation achieved from the simulation was $1.41 \mathrm{MHz}$, while the calculated FO from (6) are $1.69 \mathrm{MHz}$. The frequency spectrum of output currents are 
shown in Fig. 6. The total harmonic distortion for $I_{O 1}, I_{O 2}$ and $I_{O 3}$ are $1.13 \%, 1.18 \%$ and $1.14 \%$ respectively.

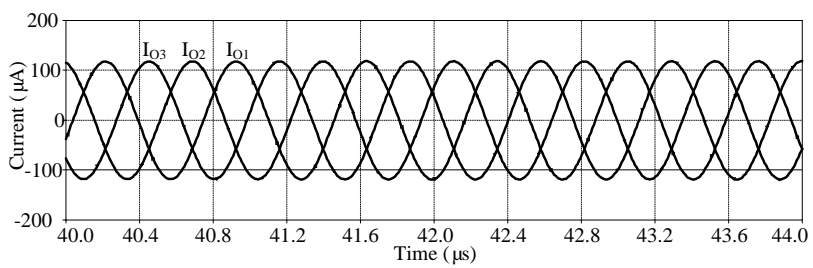

Fig. 5. Sinusoidal output waveforms.

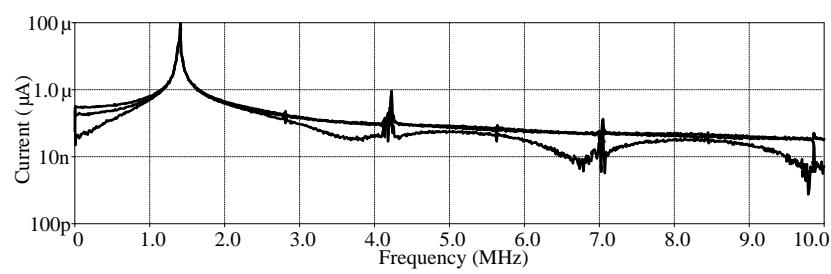

Fig. 6. Spectrum of wave forms in Fig. 5.

\section{CONCLUSION}

New current-mode multiphase sinusoidal oscillators using CCCDTA-based lossy integrators with grounded capacitors have been presented. The features of the proposed circuit are that: oscillation frequency and oscillation condition can be independently tuned; the proposed oscillator consists of merely 1 CCCDTA and 1 grounded capacitor for each phase and no additional current amplifier and availability of explicit-current outputs from high-output impedance terminals. PSPICE simulation results agree well with the theoretical anticipation.

\section{REFERENCES}

[1] J. Dunlop and D. G. Smith, Telecommunications Engineering, 3rd ed., CRC Press, 1994

[2] W. Tomasi, Electronic Communications System, New Jersey: PrenticeHall Inc., 1998.

[3] Anritsu Emea Limited. MG3700A vector signal generator datasheet. [Online]. Available: http://www.eu.anritsu.com/files/MEG3700A_ El17301.pdf.

[4] G. Eirea and S. R. Sanders, "Phase current unbalance estimation in multiphase buck converters," IEEE Transactions on Power Electronics, vol. 23, pp. 137-143, 2008.

[5] C. Toumazou, F. J. Lidgey, and D. G. Haigh, Analogue IC design: the current-mode approach, London: Peter Peregrinus, 1990.

[6] D. Biolek, "CDTA - Building block for current-mode analog signal processing," in Proc. the European Conference on Circuit Theory and Design 2003 - ECCTD'03, Krakow, Poland, 2003, pp. 397-400.

[7] M. Siripruchyanun and W. Jaikla, "CMOS current-controlled current differencing transconductance amplifier and applications to analog signal processing," International Journal of Electronics and Communications (AEU), vol. 62, pp. 277-287, 2008.

[8] M. T. Abuelma'Atti, "Current-mode multiphase oscillator using current followers" Microelectronics Journal, vol. 25, pp. 457-461, 1994.

[9] G. D. Skotis and C. Psychalinos, "Multiphase sinusodal oscillators using second generation current conveyors," Int. J. Electron. Cотти. (AEU), vol. 64, pp. 1178-1181, 2010.

[10] M. T. Abuelma'atti and M. A. Al-Qahtani, "A new current- controlled multiphase sinusoidal oscillator using translinear current conveyor," IEEE Transactions on Circuits and Systems, vol. 45, pp. $881-885,1998$
[11] C. Loescharataramdee, W. Kiranon, W. Sangpisit, and W. Yadum, "Multiphase sinusoidal oscillators using translinear current conveyors and only grounded passive components," in Proc. Southeastern Symposium on System Theory, 2004, pp. 59-63.

[12] W. Tangsrirat and W. Tanjaroen, "Current-mode multiphase sinusoidal oscillator using current differencing transconductance amplifiers," Circuits, Systems and Signal Processing, vol. 27, pp. 81- 93, 2008.

[13] W. Tangsrirat, W. Tanjaroen, and T. Pukkalanun, "Current-mode multiphase sinusoidal oscillator using CDTA-based allpass sections," International Journal of Electronics and Communications (AEU), vol. 63, pp. 616- 622, 2009

[14] W. Jaikla, M. Siripruchyanun, D. Biolek, and V. Biolkova, "High-output-impedance current-mode multiphase sinusoidal oscillator employing current differencing transconductance amplifier-based allpass filters," International Journal of Electronics, vol. 97, pp. 811-826, 2010.

[15] K. Klahan, W. Tangsrirat, and W. Surakampontorn, "Realization of multiphase sinusodal oscillator using CDBAs," in Proc. IEEE Asia-Pacific Conf. Circ. Sys., 2004, pp. 725-728.

[16] D.-S. Wu, S.-I. Liu, Y.-S. Hwang, and Y.-P. Wu, "Multiphase sinusoidal oscillator using the CFOA pole," IEE Proc. Circuits Devices Syst, vol. 142, pp. 37-40, 1995.

[17] P. Uttaphut, "New Current-mode Multiphase Sinusoidal Oscillators Based on CCCCTA-based Lossy Integrators," Electrical Review, pp. 291-295, 2012

[18] M. Kumngern, "Current-mode multiphase sinusoidal oscillator using current-controlled current differencing transconductance amplifiers," presented at 2010 IEEE International Conference of Electron Devices and Solid-State Circuits (EDSSC), 2010

[19] W. Jaikla and P. Prommee, "Electronically tunable current-mode multiphase sinusoidal oscillator employing CCCDTA-base allpass filters with only grounded passive elements," Radioengineering, vol 20, no. 3, pp. 594-599, 2011.

[20] D. R. Frey, "Log-domain filtering: an approach to current-mode filtering," IEE Proc.-G, Circuits Syst. Devices, vol. 140, pp. 406-416, 1993.

S. Siripongdee received the B.S.I. Ed. degree in electronics and computer and the M. Tech. Ed. electrical communications engineering from King Mongkut's Institute of Technology Ladkrabang (KMITL), Thailand in 1997 and 2002, respectively. He has been with Department of Engineering Education, Faculty of Industrial Education, King Mongkut's Institute of Technology Ladkrabang, Bangkok, Thailand since 1997. His research interests include electronic communications, analog signal processing and analog integrated circuit.

S. Suwanjan received the B.S.I. Ed. degree in telecommunication engineering and M. Eng in electrical engineering from King Mongkut's Institute of Technology Ladkrabang (KMITL), Thailand in 1992 and 1998, respectively. He has been with Department of Engineering Education, Faculty of Industrial Education, King Mongkut's Institute of Technology Ladkrabang, Bangkok, Thailand since 1992. His research interests include electronic communications, analog signal processing and analog integrated circuit.

S. Tuntrakool received the B.S.I. Ed. degree in telecommunication engineering from King Mongkut's Institute of Technology Ladkrabang (KMITL), Thailand in 1993, and M.S. in electrical engineering from Vanderbilt University, USA in 2004. He has been with Department of Engineering Education, Faculty of Industrial Education, King Mongkut's Institute of Technology Ladkrabang, Bangkok, Thailand since 1993. His research interests include electronic communications, analog signal processing and analog integrated circuit.

W. Jaikla received the B.S.I. Ed. degree in telecommunication engineering from King Mongkut's Institute of Technology Ladkrabang (KMITL), Bangkok, Thailand in 2002, M. Tech. Ed. in electrical technology and Ph.D. in electrical education from King Mongkut's University of Technology North Bangkok (KMUTNB) in 2004 and 2010, respectively. From 2004 to 2011 he was with Electric and Electronic Program. 